\title{
Functional dissection and module swapping of fungal cyclooligomer depsipeptide synthetases $\uparrow$
}

\author{
Dayu Yu, ${ }^{a, b, c}$ Fuchao Xu, ${ }^{a, b, c}$ David Gage ${ }^{b}$ and Jixun Zhan*b \\ Received (in XXX, XXX) Xth XXXXXXXXX 2013, Accepted Xth XXXXXXXXX 2013 \\ ${ }_{5}$ DOI: $10.1039 / b 000000 x$
}

BbBSLS and BbBEAS were dissected and reconstituted in Saccharomyces cerevisiae. The intermodular linker is essential for the reconstitution of the separate modules. Module 1 can 10 be swapped between BbBEAS and BbBSLS, while modules 2 and 3 control the product profiles. BbBSLS is a flexible enzyme that also synthesizes beauvericins.

Beauvericin, beauvericins A-C and bassianolide (Fig. 1A) are a group of promising anticancer cyclooligomer depsipeptides 15 (CODs) from several fungal species. ${ }^{1}$ The genes encoding the synthetases for these compounds have been reported. ${ }^{2,3}$ The beauvericin synthetase (BbBEAS, $352 \mathrm{kDa}$ ) and bassianolide synthetase (BbBSLS, $348 \mathrm{kDa}$ ) from Beauveria bassiana ATCC 7159 have the same domain organization of C1-A1-T1-C2-A220 MT-T2a-T2b-C3 (Fig. 1B) and share 66\% identity and 79\% similarity overall. Most reported fungal COD synthetases (CODSs) have the same structural organization except a bassianolide synthetase from Xylaria sp. BCC1067 that has an additional C-terminal reductase (R) domain. ${ }^{3}$

${ }_{25}$ The assembly of fungal CODs has been previously proposed. ${ }^{4}$ CODSs take a unit of D-hydroxycarboxylic acid and L-amino acid to form the corresponding dipeptidols. These intermediates will then undergo catalytic cyclooligomerization to form the final CODs. BbBEAS synthesizes cyclohexadepsipeptides including 30 beauvericin and its congeners beauvericins A-C, while BbBSLS uses D-hydroxyisovaleric acid (D-Hiv) and L-leucine (L-Leu) to generate the cyclohexadepsipeptide bassianolide. We have recently successfully reconstituted BbBEAS and BbBSLS in the intact form in Saccharomyces cerevisiae BJ5464-NpgA and 35 achieved high-titer production of beauvericins and bassianolide. ${ }^{5}$ In this paper, we report the functional dissection and reconstitution of these modular enzymes in $S$. cerevisiae, characterization of BbBSLS as a flexible enzyme that synthesizes both bassianolide and beauvericins, module swapping between ${ }_{40}$ BbBEAS and BbBSLS, and construction of hybrid CODSs.

The boundary of BbBEAS and BbBSLS between M1 and M2 was analyzed by multiple sequence alignment of nonribosomal

\footnotetext{
${ }^{a}$ Department of Applied Chemistry and Biological Engineering, College of Chemical Engineering, Northeast Dianli University, Jilin, Jilin 132012, China.

${ }^{b}$ Department of Biological Engineering, Utah State University, 4105 Old Main Hill, Logan, UT 84322, USA. Fax: +1 435797 1248; Tel: +1 435797 8774; E-mail: jixun.zhan@usu.edu

${ }^{c}$ These authors contributed equally to this work.

$\dagger$ Electronic Supplementary Information (ESI) available: Primers, plasmids, and experimental detals. See DOI: 10.1039/b000000x/
}

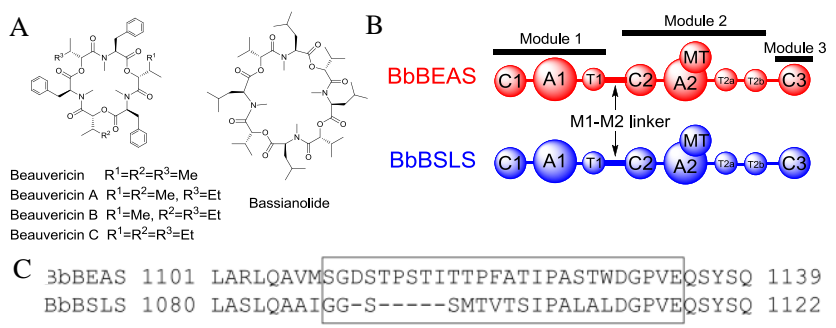

Fig. 1 (A) Structures of beauvericins and bassianolide. (B) Domain organization of BbBEAS and BbBSLS. C: condensation domain; A: adenylation domain; T: thiolation domain; MT: methyltransferase domain. Different colors (red for BbBEAS and blue for BbBSLS) are used to differentiate these two similar CODSs. (C) Partial alignment of the amino acid sequences of BbBEAS and BbBSLS. The M1-M2 linker is boxed.

peptide synthetases (NRPSs) (Figs. S1 and S2) and a web-based software tool. ${ }^{6}$ The intermodular linker between M1 and M2 45 (M1-M2 linker, abbreviated as "linker" hereafter) is shown in Figs. 1B and 1C. We used two Escherichia coli/S. cerevisiae shuttle vectors, pXW55 and pXW06, to express the dissected enzymes in S. cerevisiae BJ5464-NpgA. ${ }^{7}$ The primers used and plasmids constructed in this study are shown in Tables S1 and S2, 50 respectively $(\mathrm{ESI} \dagger)$. The first two expression plasmids for the dissection of BbBEAS were pDY45 and pDY58, which harbor the linker-containing BbBEAS fragments bbBeasM1- and bbBeasM23-, respectively (Fig. 2). Expression of either of these plasmids in the yeast yielded no products. Co-expression of these 55 two plasmids in S. cerevisiae BJ5464-NpgA led to the production of beauvericins, including beauvericin and beauvericins A-C (ESI-MS spectra are shown in Fig. S3), at $10.67 \pm 1.44 \mathrm{mg} \mathrm{L}^{-1}$ (Fig. 2). Although the titer is lower than that of the intact enzyme $\left(33.82 \pm 1.41 \mathrm{mg} \mathrm{L}^{-1}\right)$, this result for the first time demonstrated 60 that BbBEAS can be dissected into modules and functionally reconstituted in the yeast. To examine whether the intermodular linker influences the interaction and function of the modules, we also cloned the linker-lacking bbBeasM1 and bbBeasM23 to construct two additional expression plasmids pDY99 and pDY98. ${ }_{65}$ Co-expression of these two plasmids in the yeast yielded no products (Fig. 2), suggesting that the linker is essential for the reconstitution of beauvericin biosynthesis by the dissected fragments. We further co-expressed pDY45 with pDY98 and pDY58 with pDY99. Interestingly, the former did not yield any 70 products, while the latter generated beauvericins in a much lower titer (Fig. 2). These results suggested that the N-terminal linker of BbBEASM23 is required for either the stability of the fragment 


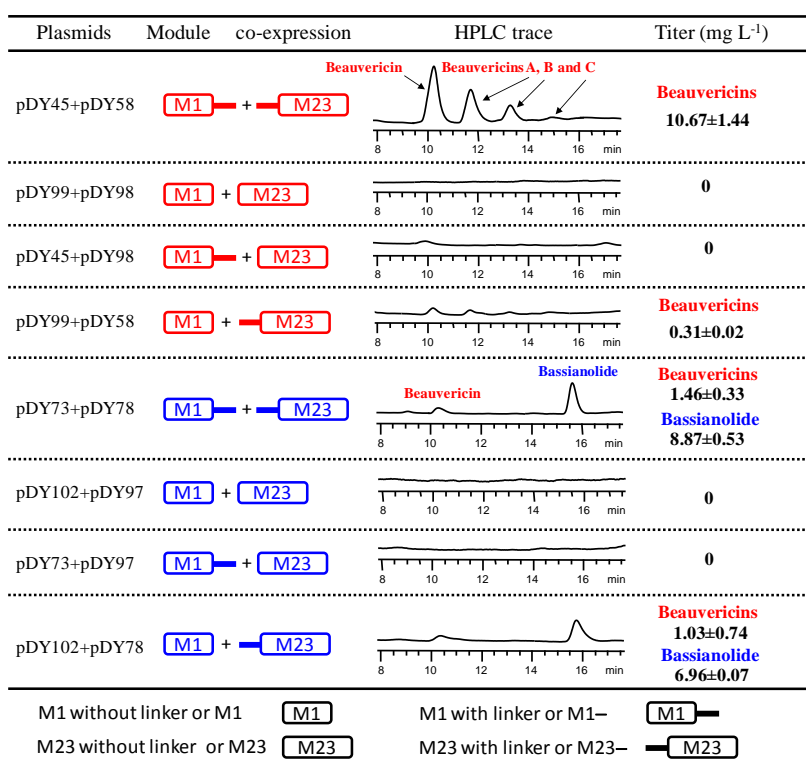

Fig. 2 Dissection and reconstitution of BbBEAS and BbBSLS in $S$. cerevisiae BJ5464-NpgA. BbBEAS and BbBSLS were dissected into two fragments, M1 and M23, with or without the liner. The fragments were co-expressed in S. cerevisiae BJ5464-NpgA and the production of beauvericins and bassianolide was analyzed by LC-MS.

of modules 2 and 3 (M23) or the interaction between M1 and M23. Inclusion of the same linker in the C-terminus of BbBEASM1 significantly improved the collaborative work of M1 and M23, as reflected by the increased titer of beauvericins.

5 Using a similar approach, we dissected BbBSLS into M1 and M23 (with or without the linker) by constructing four plasmids pDY73, pDY78, pDY97 and pDY102 (Table S2, ESI $\dagger$ ). Coexpression of pDY73 and pDY78, which harbor BbBSLSM1and BbBSLSM23-, respectively, reconstituted the production of 10 bassianolide (ESI-MS spectrum is shown in Fig. S3) in the yeast at $8.87 \pm 0.53 \mathrm{mg} \mathrm{L}^{-1}$ (Fig. 2). Unexpectedly, beauvericin and trace amounts of beauvericins A-C were also produced by this strain. To find out whether the production of beauvericins were due to the dissection of BbBSLS, which may have influenced the overall 15 structure of the modular enzyme, we re-examined the product profile of S. cerevisiae BJ5464-NpgA/pDY42. LC-MS analysis revealed that the intact BbBSLS also produced beauvericins (Fig. 3 ) in addition to bassianolide. In contrast, the yeast strain expressing the intact BbBEAS only produced beauvericins (Fig. 20 3). This indicated that BbBSLS is a flexible CODS which can incorporate either L-Phe or L-Leu and catalyzes trimerization or tetramerization of the monomers. We also searched for the potential hybrid products containing both $N$-methyl-L-Leu and $N$ methyl-L-Phe by extracting the target ion peaks from the ESI-MS 25 spectra. However, no hybrid CODs were found. We propose that bassianolide as the major product perfectly fits the active site of BbBSLS. The trimeric beauvericins containing $N$-methyl-L-Phe which is larger than $N$-methyl-L-Leu may occupy similar space as the tetramer bassianolide does. The possible hybrid products may 30 not fit the active site well and thus cannot be synthesized efficiently to be detected. In addition, the condensation of the monomers in COD biosynthesis is not well understood, and it is also likely that missing of the hybrid CODs is due to insufficient capacity of the domains involved in the oligomerization to

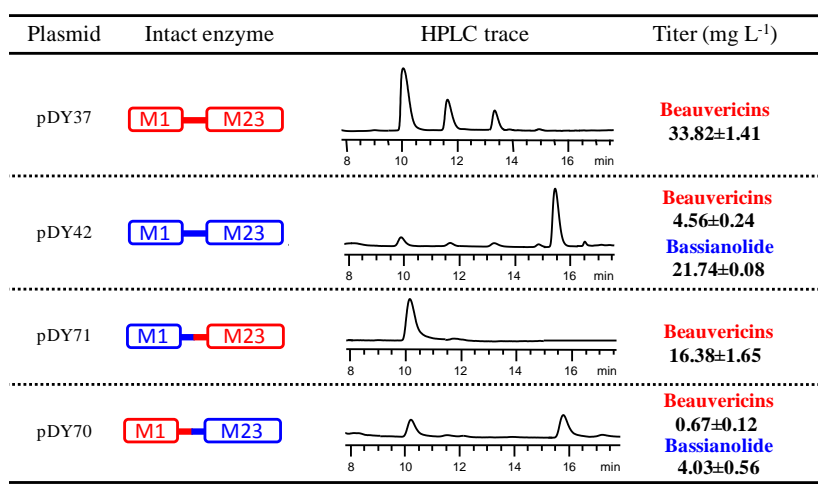

Fig. 3 Product profiles of the original and hybrid CODSs in the intact form. Both wild type CODSs (pDY37: BbBEAS; pDY42: BbBSLS) and hybrid enzymes (pDY71: BbBSLSM1-BbBEASM23; pDY70: BbBEASM1-BbBSLSM23) were expressed in S. cerevisiae BJ5464-NpgA and the production of CODs was analyzed by LC-MS

35 incorporate monomers containing different amino acid precursors into the products. When pDY102 and pDY97, which respectively harbor the linker-lacking M1 and M23 of BbBSLS, were coexpressed in the yeast, no products were detected. This is consistent with the result for BbBEAS. Co-expression of pDY73 40 with pDY97 and pDY102 with pDY78 yielded similar results to those for BbBEAS as well (Fig. 2).

Functional dissection of BbBEAS and BbBSLS provides a useful tool for further investigation of these giant enzymes. Expression of truncated fragments of the enniatin synthetase from ${ }_{45}$ Fusarium scirpi has been previously attempted in E. coli. ${ }^{8}$ However, the proteins were mainly produced as inclusion body and required renaturation for testing their adenylation activity. No CODs have ever been synthesized using these fragments. In this work, we dissected two CODSs into functional modules and 50 reconstituted the production of the corresponding products by coexpression of the modules with the important intermodular linker. This opens a new opportunity to investigate and engineer the functional modules instead of the giant enzymes for combinatorial biosynthesis of new bioactive molecules.

55 The primary function of BbBEASM1 and BbBSLSM1 is supposed to activate and load D-Hiv onto the CODSs, although we have recently found that BbBEASM1 can also utilize D-Hmv to synthesize beauvericins A-C. ${ }^{5}$ We hypothesize that the M1 from BbBEAS and BbBSLS can be exchanged because of the 60 similar function. To test this hypothesis, we made eight cotransformations using the eight expression plasmids constructed above. As shown in Fig. 4, when BbBSLSM1- and BbBEASM23- were co-expressed in the yeast, beauvericins were synthesized. Similarly, co-expression of BbBEASM1- and ${ }_{65}$ BbBSLSM23- led to the production of bassianolide and beauvericins. These results clearly indicated that M1 of BbBEAS and BbBSLS can be exchanged without influencing the product profiles. The final products are determined by M23, which controls the selection of the L-amino acid, forms the 70 corresponding dipeptidol, and catalyzes the subsequent cyclooligomerization. Additional module swapping experiments that contained different combinations of heterologous M1 and M23, with or without the linker, were conducted to further test the role of the M1-M2 linker in the collaborative work between 75 heterologous modules. As shown in Fig. 4, any co-transformants 
containing the linker-lacking BbBEASM23 or BbBSLSM23 yielded no products. Combinations of a linker-lacking M1 with a heterologous linker-containing M23 afforded products corresponding to the M23 (Fig. 4) in relatively low titers. These 5 results indicated that the N-terminal linker of M23 of BbBEAS and BbBSLS is required for the collaborative work of heterologous M1 and M23. It has been previously reported that short communication-mediating (COM) domains play decisive role in protein-protein recognition in bacterial modular NRPSs

10 such as those in tyrocidine biosynthesis. ${ }^{9}$ Similarly, intermodular linkers in polyketide synthases are involved in the assembly of functional modules and the intermodular polyketide chain transfer. ${ }^{10}$ Our results revealed that a similar role of the intermodular linker in fungal CODSs. We propose that the $\mathrm{C} 2$ 15 domain has specific binding sites for the donor and acceptor that will allow these two thiolated precursors, which are respectively linked to $\mathrm{T} 1$ and $\mathrm{T} 2$, to recognize and bind to $\mathrm{C} 2$, as seen in other NRPSs such as the gramicidin synthetase. ${ }^{11}$

In addition to testing module swapping of CODSs using 20 dissected enzymes, we also made two hybrid enzymes BbBEASM1-BbBSLSM23 and BbBSLSM1-BbBEASM23. The linkers between the heterologous M1 and M23 were made up of the C-terminal portion of the linker of $\mathrm{M} 1$ and $\mathrm{N}$-terminal portion of the linker of M23 in the original enzymes. As shown in Fig. 3, 25 LC-MS analysis revealed the biosynthesis of beauvericins in $S$. cerevisiae BJ5464-NpgA/pDY71 and the production of bassianolide and beauvericins in $S$. cerevisiae BJ5464$\mathrm{NpgA} / \mathrm{pDY} 70$. These results were consistent with those from the co-expression of the separate modules. These same results from 30 both intact enzymes and co-expressed dissected enzymes further confirmed that BbBEASM1 and BbBSLSM1 can be swapped.

Although module/domain swapping represents an attractive tool to create new molecules, it is often hampered by the heavily impaired biosynthetic capacity of the chimeric NRPSs. ${ }^{12}$ ${ }_{35}$ Successful construction of hybrid CODSs in the intact form indicated that these fungal enzymes are friendly to genetic manipulations. There were several reports on the construction of bacterial hybrid NRPSs. For example, hybrid NRPSs were made by fusions of modules from TycA, TycB and TycC involved in 40 tyrocidine biosynthesis. ${ }^{13}$ Another paper has reported the construction of a new bi-modular NRPS by replacing an activation domain with one with $N$-methylation activity using the actinomycin NRPS. ${ }^{14}$ While these enzymes were made of modules from the same biosynthetic systems, other studies on 45 intermodular linkers/COM domains have allowed the crosstalk between modules from different NRPSs. ${ }^{9}$ However, no moduleswapping work on fungal NRPSs has been reported. This work thus represents the first example of construction of functional hybrid fungal CODSs, which provides a comprehensive platform 50 for future engineering of fungal CODSs in both intact and dissected forms for novel "unnatural" natural products.

In summary, this work revealed that modular CODSs from fungi can be functionally dissected into modules and expressed in $S$. cerevisiae. Co-expression of the dissected M1 and M23 of 55 these CODSs reconstituted the production of their products. The linker between $\mathrm{M} 1$ and M2 plays a pivotal role in the reconstitution of the dissected CODSs. BbBEASM1 and BbBSLSM1 are exchangeable and the final products are determined by M23. BbBSLS is a flexible CODS that synthesizes 60 both bassianolide and beauvericins.

This research was supported by a Utah State University Seed Program to Advance Research Collaborations (SPARC) grant and a grant from the National Natural Science Foundation of China

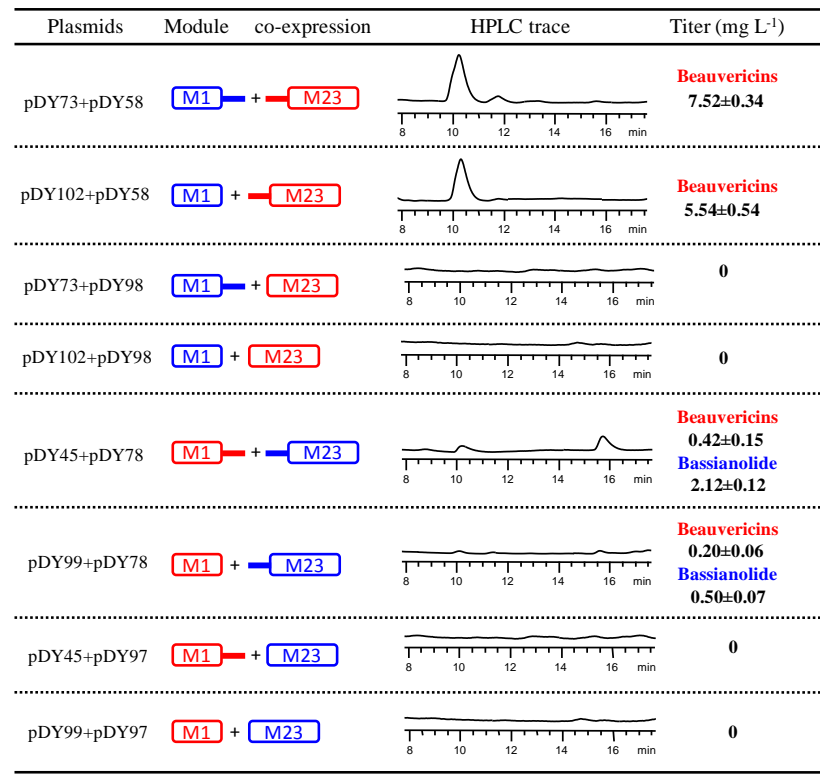

Fig. 4 Module swapping between BbBEAS and BbBSLS. Heterologous modules from BbBEAS and BbBSLS were co-expressed in S. cerevisiae BJ5464-NpgA and the products were analyzed by LC-MS.

\section{(31170763).}

\section{${ }_{65}$ Notes and references}

1 (a) J. Zhan, A. Burns, M. X. Liu, S. H. Faeth and A. A. L. Gunatilaka, J. Nat. Prod., 2007, 70, 227-232; (b) A. Logrieco, A. Moretti, G. Castella, M. Kostecki, P. Golinski, A. Ritieni and J. Chelkowski, Appl. Environ. Microbiol., 1998, 64, 3084-3088; (c) Y. Xu, J. Zhan, E. M. K. Wijeratne, A. Burns, A. A. L. Gunatilaka and I. Molnár, J. Nat. Prod., 2007, 70, 1467-1471.

2 (a) Y. Xu, R. Orozco, E. M. K. Wijeratne, A. A. L. Gunatilaka, S. P. Stock and I. Molnár, Chem. Biol., 2008, 15, 898-907; (b) Y. Xu, R. Orozco, E. M. K. Wijeratne, P. Espinosa-Artiles, A. A. L. Gunatilaka, S. P. Stock and I. Molnár, Fungal Genet. Biol., 2009, 46, 353-364.

3 J. Jirakkakul, J. Punya, S. Pongpattanakitshote, P. Paungmoung, N. Vorapreeda, A. Tachaleat, C. Klomnara, M. Tanticharoen and S. Cheevadhanarak, Microbiology, 2008, 154, 995-1006.

804 R. Süssmuth, J. Müller, H. von Döhren and I. Molnár, Nat. Prod. Rep., 2011, 28, 99-124.

5 D. Yu, F. Xu, J. Zi, S. Wang, D. Gage, J. Zeng and J. Zhan, Metab. Eng., 2013, http://dx.doi.org/10.1016/j.ymben.2013.1004.1001.

6 M. Z. Ansari, G. Yadav, R. S. Gokhale and D. Mohanty, Nucleic Acids Res., 2004, 32, W405-W413.

7 S. M. Ma, J. W. H. Li, J. W. Choi, H. Zhou, K. K. M. Lee, V. A. Moorthie, X. Xie, J. T. Kealey, N. A. Da Silva, J. C. Vederas and Y. Tang, Science, 2009, 326, 589-592.

8 A. Haese, R. Pieper, T. Vonostrowski and R. Zocher, J. Mol. Biol., 1994, 243, 116-122.

9 (a) M. Hahn and T. Stachelhaus, Proc. Natl. Acad. Sci. U. S. A., 2006, 103, 275-280; (b) M. Hahn and T. Stachelhaus, Proc. Natl. Acad. Sci. U. S. A., 2004, 101, 15585-15590; (c) V. Siewers, R. San-Bento and J. Nielsen, Biotechnol. Bioeng., 106, 841-844.

9510 R. S. Gokhale, S. Y. Tsuji, D. E. Cane and C. Khosla, Science, 1999, 284, 482-485.

11 P. J. Belshaw, C. T. Walsh and T. Stachelhaus, Science, 1999, 284, 486-489.

12 M. A. Fischbach, J. R. Lai, E. D. Roche, C. T. Walsh and D. R. Liu, Proc. Natl. Acad. Sci. U. S. A., 2007, 104, 11951-11956.

13 H. D. Mootz, D. Schwarzer and M. A. Marahiel, Proc. Natl. Acad. Sci. U. S. A., 2000, 97, 5848-5853.

14 F. Schauwecker, F. Pfennig, N. Grammel and U. Keller, Chem. Biol., 2000, 7, 287-297. 